\title{
Hepatic sarcoidosis: Clinical characteristics and outcome
}

\section{Authors}

Christiana Graf, Jeannette Arncken, Christian M. Lange, Katharina Willuweit, Jörn M. Schattenberg, Jessica Seessle, Julia Lang-Meli, Tobias Böttler, Julia Dietz, Nils Wetzstein, Antonia Mondorf, Johannes Vermehren, Gernot Rohde, Stefan Zeuzem, Anita Pathil

\section{Correspondence}

christiana.graf@kgu.de (C. Graf).

\section{Graphical abstract}

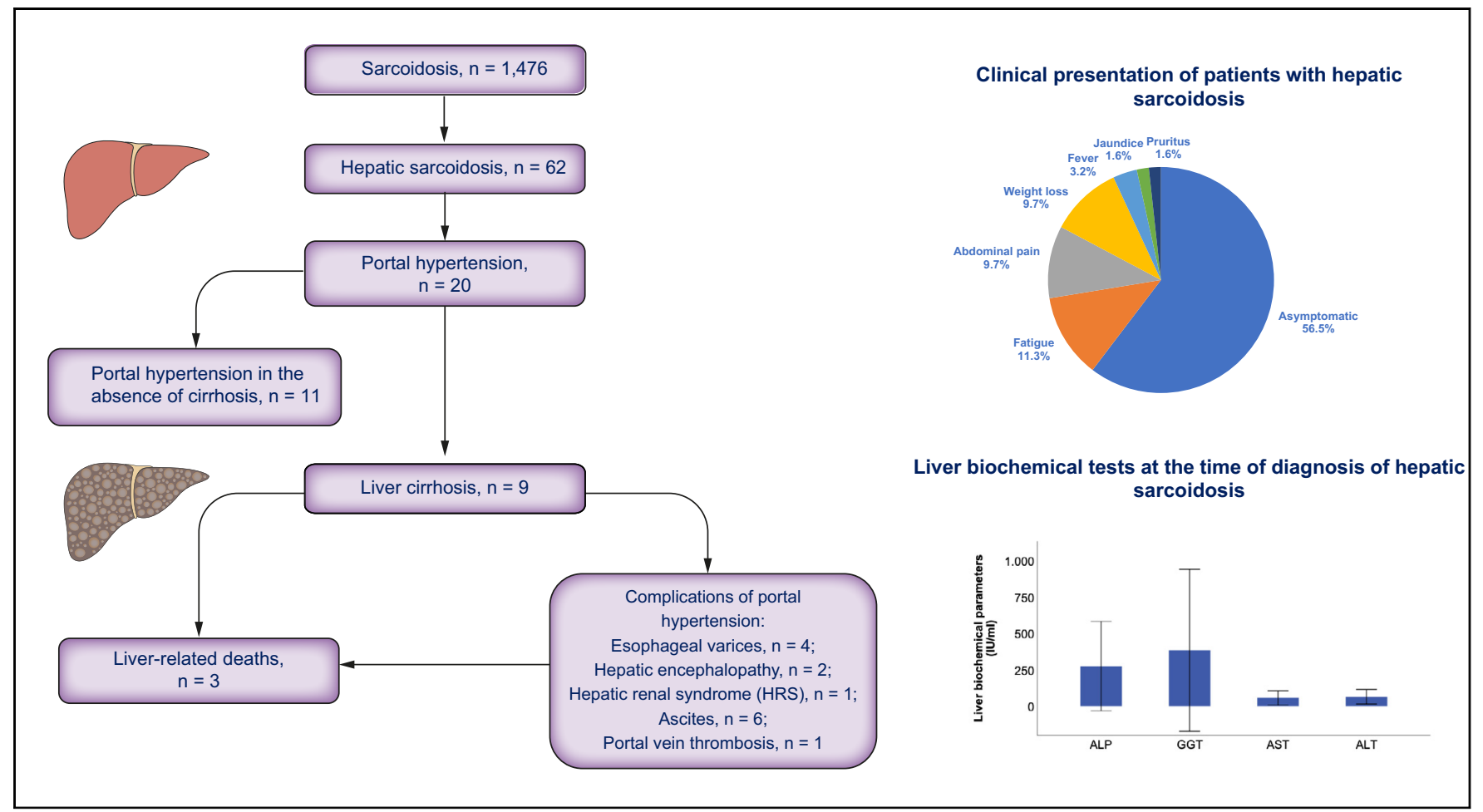

\section{Highlights}

- There has been little research on clinical characteristics and the clinical outcome of patients with hepatic sarcoidosis.

- One-third of patients with hepatic sarcoidosis presented with clinically significant portal hypertension, and $14.5 \%$ suffered from cirrhosis.

- Biochemically, a cholestatic pattern of liver enzyme elevations was the most common abnormality.

- The correct and early identification of hepatic involvement in sarcoidosis is crucial because of the potentially progressive course of disease.
Lay summary

Clinical diagnostic and surveillance of hepatic involvement in sarcoidosis has not been standardised, and management of hepatic involvement is a clinical challenge, since it remains poorly characterised in many ways. Our results show that one-third of patients with hepatic sarcoidosis presented with clinically significant portal hypertension, $14.5 \%$ suffered from cirrhosis, and 3 patients died owing to liver-related complications. Regarding pharmacological treatment options, corticosteroids and UDCA were the medical agents most frequently used, and both of them have been shown to induce biochemical response in the majority of patients. These findings highlight the importance of correctly and early identifying hepatic involvement in sarcoidosis, because of the potentially progressive course of disease. 


\title{
Hepatic sarcoidosis: Clinical characteristics and outcome
}

\author{
Christiana Graf, ${ }^{1, *}$ Jeannette Arncken, ${ }^{1}$ Christian M. Lange, ${ }^{2}$ Katharina Willuweit, ${ }^{2}$ Jörn M. Schattenberg, ${ }^{3}$ \\ Jessica Seessle, ${ }^{4}$ Julia Lang-Meli, ${ }^{5}$ Tobias Böttler, ${ }^{5}$ Julia Dietz, ${ }^{1}$ Nils Wetzstein, ${ }^{6}$ Antonia Mondorf, \\ Johannes Vermehren, ${ }^{1}$ Gernot Rohde, ${ }^{1}$ Stefan Zeuzem, ${ }^{1}$ Anita Pathil ${ }^{1}$

\begin{abstract}
${ }^{1}$ Department of Internal Medicine I, University Hospital Frankfurt, Frankfurt am Main, Germany; ${ }^{2}$ Department of Gastroenterology and Hepatology, University Hospital Essen, Essen, Germany; ${ }^{3}$ Department of Internal Medicine I, University Medical Center Mainz, Mainz, Germany; ${ }^{4}$ Department of Internal Medicine IV, University of Heidelberg, Heidelberg, Germany; ${ }^{5}$ Department of Medicine II, University Hospital Freiburg, Freiburg, Germany; ${ }^{6}$ Department of Infectious Diseases, University Hospital Frankfurt, Frankfurt, Germany
\end{abstract}

JHEP Reports 2021. https://doi.org/10.1016/j.jhepr.2021.100360

Background \& Aims: Clinical manifestation of hepatic involvement in sarcoidosis can vary from asymptomatic disease to severe complications such as cirrhosis and portal hypertension. However, data on hepatic sarcoidosis are limited, and evidence-based recommendations are lacking. Our study aimed to assess the features and clinical course of hepatic sarcoidosis in a predominantly Caucasian cohort.

Methods: We performed a retrospective study including all patients with hepatic sarcoidosis between 2004 and 2020 in 5 German centres. The median follow-up time was 36 months (range 0.0-195). Data on demographic parameters, clinical manifestations, diagnostic test results, treatment, and outcome were collected.

Results: A total of 1,476 patients with sarcoidosis and 62 patients with hepatic involvement (4.2\%) were identified. Of the patients, $51.6 \%$ were female, and $80.6 \%$ were Caucasian. Most patients were asymptomatic and were observed to have a cholestatic pattern of liver enzyme elevations. Cirrhosis was detected in 9 patients (14.5\%), of whom 6 developed clinical manifestations of portal hypertension. Fifty-four patients were medically treated, most commonly with glucocorticoids (69.4\%) or ursodeoxycholic acid (UDCA) (40.3\%). Levels of alkaline phosphatase (ALP) decreased by $60.8 \%$ on average from baseline in patients treated with glucocorticoids and by $59.9 \%$ in patients treated with UDCA. Seventeen patients received treatment augmentation with a second line agent, of whom 8 patients normalised ALP levels during follow-up. None of the patients underwent liver transplantation or developed hepatocellular carcinoma (HCC). Three of the patients died during follow-up owing to liver-related complications.

Conclusions: Hepatic involvement in sarcoidosis was found in $4.2 \%$ of patients with sarcoidosis and was clinically significant in $14.5 \%$ of those. These findings highlight the importance of early identifying, monitoring, and treating hepatic sarcoidosis, given its increased mortality when associated with end-stage liver disease.

Lay summary: Clinical diagnostic and surveillance of hepatic involvement in sarcoidosis has not been standardised, and management of hepatic involvement is a clinical challenge, since it remains poorly characterised in many ways. Our results show that one-third of patients with hepatic sarcoidosis presented with clinically significant portal hypertension, $14.5 \%$ suffered from cirrhosis, and 3 patients died owing to liver-related complications. Regarding pharmacological treatment options, corticosteroids and UDCA were the medical agents most frequently used, and both of them have been shown to induce biochemical response in the majority of patients. These findings highlight the importance of correctly and early identifying hepatic involvement in sarcoidosis, because of the potentially progressive course of disease.

(c) 2021 The Authors. Published by Elsevier B.V. on behalf of European Association for the Study of the Liver (EASL). This is an open access article under the CC BY-NC-ND license (http://creativecommons.org/licenses/by-nc-nd/4.0/).

\section{Introduction}

Sarcoidosis is a chronic multisystemic granulomatous disease of unknown aetiology, which is characterised by the formation of noncaseating granulomas and can affect multiple organs and tissues. Sarcoidosis occurs worldwide; however, its prevalence is

Keywords: Hepatic sarcoidosis; Outcome; Treatment; Hepatic granuloma; Liver involvement.

Received 6 May 2021; received in revised form 13 August 2021; accepted 25 August 2021 available online 3 September 2021

* Corresponding author. Address: Medizinische Klinik 1, Universitätsklinikum Frankfurt am Main, Theodor-Stern-Kai 7, 60590 Frankfurt am Main, Germany. Tel.: +4969-6301-5122. Fax: +4969-6301-83112.

E-mail address: christiana.graf@kgu.de (C. Graf). reported to be twice as high in Nordic countries and among African Americans. ${ }^{1}$ In contrast, low rates have been reported in patients of Chinese and Taiwanese origin. ${ }^{2}$ Females are affected more than males across all ages and ethnicities, with an age peak at diagnosis of $20-40$ years. $^{3}$

Although sarcoidosis can affect virtually every organ of the body, the pulmonary system is the most common site of involvement, which is affected in about $90 \%$ of cases and has been the greatest subject of intense research so far. ${ }^{4}$ Other organs often affected are the liver, skin, lymph node, and eye. Liver involvement in sarcoidosis was found in about $50-65 \%$ of cases. ${ }^{5}$ However, post-mortem studies based on autopsy reported higher rates compared with antemortem studies. ${ }^{6}$ Thus, prevalence may 
be considerably higher, and liver involvement by sarcoidosis might be under-recognised in clinical practice owing to the relatively symptomless nature of the disease. Despite its asymptomatic course, a minority of patients progress to chronic cholestatic disease, portal hypertension, Budd-Chiari syndrome, and cirrhosis, which may require liver transplantation. Medical treatment options of hepatic sarcoidosis such as glucocorticoids or immunomodulators exist, but they are still not well defined as to whether they can prevent progression of the disease or are just able to alleviate symptoms. Moreover, little is known about how to clinically manage these patients in the best way and whether there are potential biomarkers or further diagnostic factors that reliably predict the course of disease.

All in all, surveillance of hepatic involvement in sarcoidosis has not been standardised and management of hepatic involvement is a clinical challenge, given that it remains poorly characterised in many ways and the current textbook knowledge is mainly based on clinical experience and retrospective case studies. This lack of published data is particularly striking, because its worldwide prevalence is similar to the prevalence of other, well-characterised liver disorders (e.g. Wilson disease or $\alpha 1$-antitrypsin deficiency). ${ }^{7-9}$ The goal of our following study was to better characterise the epidemiology and clinical course of hepatic sarcoidosis as well as to evaluate the therapy options used.

\section{Patients and methods \\ Study population}

In this retrospective study, adult patients aged 18 years and older with hepatic sarcoidosis who attended the participating centres (University Hospital Frankfurt, University Hospital Essen, University Medical Center Mainz, University Hospital Heidelberg, and University Hospital Freiburg) between 1 September 2004 and 1 March 2020 were included in the study. Possible patients were identified by systematically searching the patient chart database of the participating hospitals for sarcoidosis-related diagnosis codes D86.0, D86.1, D86.2, D86.3, D86.8, and D86.9 of the International Classification of Diseases, Tenth Revision (ICD10), German Modification.

Medical records of the resulting cases were systematically reviewed. The diagnosis of sarcoidosis required a diagnosis made by physicians who evaluated the patient. According to the American Thoracic Society (ATS) guidelines, the diagnosis of sarcoidosis relied on 3 major criteria: (i) a compatible clinical presentation, (ii) the presence of noncaseating granuloma on histopathology, and (iii) the exclusion of other granulomatous diseases such as tuberculosis and fungal infection. ${ }^{10}$ Stage I pulmonary sarcoidosis, which could be diagnosed by the presence of symmetric bilateral hilar adenopathy after excluding other possible causes, was the only case that required histopathologic confirmation of liver involvement.

Confirmed diagnoses of sarcoidosis were examined for hepatic involvement. To confirm the diagnosis of hepatic sarcoidosis, we collected data on hepatic lesions detected in imaging studies, characteristics of hepatic granulomas in biopsies, clinical and/or laboratory evidence of liver involvement, and extrahepatic manifestations of systemic sarcoidosis. In cases of systemic sarcoidal involvement with histopathologic confirmation in at least 1 affected organ, the diagnosis of hepatic involvement was defined by the presence of hypodense nodular lesions in abdominal imaging and by abnormal biochemical parameters without other identifiable causes (such as viral hepatitis, autoimmune liver diseases, fatty liver, drugs, and significant alcohol consumption). Biopsy-proven hepatic sarcoidosis was not necessary in such cases. However, if patients had isolated hepatic manifestation, histopathological confirmation of sarcoidosis and exclusion of other causes of granulomatous hepatitis were required (such as tuberculosis, drugs, and primary biliary cirrhosis). Cases that were excluded from further analysis concerned concomitant hepatic diseases such as fatty liver disease, significant alcohol consumption ( $>20 \mathrm{~g} /$ day for women and $>30$ $\mathrm{g}$ /day for men), viral hepatitis, and storage and autoimmune liver diseases, which were diagnosed by patient history, laboratory workup, imaging, and histopathological findings. Moreover, patients with an active cancer disease, pregnant women, and patients aged 17 years and younger were also excluded. In all cases, the presence of fibrosis and cirrhosis was assessed histologically.

Extracted patients' characteristics included sex, age, ethnicity, and BMI at baseline. In addition, liver biochemical tests, imaging study of the liver, histopathological results, clinical manifestation, medical therapies used, and liver-related complications were recorded at baseline and at yearly follow-up. This study was approved by the local ethics committee of the Goethe University Frankfurt (ethics committee reference number: 20-711) and the involved partners. Owing to the retrospective, anonymous, and noninterventional nature of this study, no informed consent from individual patients had to be obtained.

\section{Statistical analysis}

Statistical analyses were performed using IBM SPSS 26.0 statistical software package (SPSS/IBM, Munich, Germany). Characteristics of the cohort were examined by descriptive statistics (percentages, means, SD, etc.). Comparisons between 2 groups with quantitative variables were performed using the Wilcoxon signed rank test. Associations between categorial variables were tested by Spearman's correlation coefficients and their associated probability. All tests were 2-sided, and a $p$ value of less than 0.05 was judged to be statistically significant.

\section{Results}

\section{Baseline and clinical characteristics}

The main baseline patient characteristics of the overall study population, including demographic and clinical features, are listed in Table 1. Among 1,476 identified patients with sarcoidosis, 62 (4.2\%) were diagnosed with liver involvement: the majority of patients was diagnosed based on histopathological findings, positive imaging study, and cholestatic liver enzyme abnormalities (47 cases), followed by positive imaging study and cholestatic liver enzyme abnormalities ( 7 cases), histopathological findings and positive imaging study (2 cases), and liver biopsy and cholestatic biochemical liver tests (6 cases).

Follow-up was available in 58 patients. Median duration of follow-up from diagnosis of hepatic sarcoidosis to last follow-up was 36 months (range $0.0-195$ ).

In our cohort of 62 patients with sarcoidal hepatic involvement, $32(51.6 \%)$ were female, $50(80.6 \%)$ were Caucasian, 3 (4.8\%) were African, and $2(3.2 \%)$ were Hispanic. Median age at diagnosis of hepatic sarcoidosis was 51.6 years (range 23.0-79.0 years).

Most cases of hepatic manifestation occurred in conjunction with pulmonary sarcoidosis $(50.0 \%)$, followed by lymphatic (38.7\%), splenic (17.7\%), ocular (9.7\%), and parotid (8.1\%) 
Table 1. Baseline clinical characteristics of patients with hepatic sarcoidosis.

\begin{tabular}{|c|c|}
\hline Characteristics & Patients $(n=62)$ \\
\hline Patient age at diagnosis (years), mean \pm SD & $51.6 \pm 12.1$ \\
\hline Female sex, $\mathrm{n}(\%)$ & $32(51.6)$ \\
\hline \multicolumn{2}{|l|}{ Ethnicity, n (\%) } \\
\hline Caucasian & $50(80.6)$ \\
\hline African American & $3(4.8)$ \\
\hline Hispanic & $2(3.2)$ \\
\hline Other & $7(11.3)$ \\
\hline BMI $\left(\mathrm{kg} / \mathrm{m}^{2}\right)$, mean $\pm \mathrm{SD}$ & $25.0 \pm 4.5$ \\
\hline Length of follow-up (years), median (range) & $3.0(0.0-16.25)$ \\
\hline \multicolumn{2}{|l|}{ Extrahepatic involvement, $\mathrm{n}(\%)^{*}$} \\
\hline Pulmonary & $31(50.0)$ \\
\hline Lymphatic & $24(38.7)$ \\
\hline Splenic & $11(17.7)$ \\
\hline Ocular & $6(9.7)$ \\
\hline Parotid & $5(8.1)$ \\
\hline \multicolumn{2}{|l|}{ Clinical presentation, $\mathrm{n}(\%)^{\dagger}$} \\
\hline Asymptomatic & $35(56.5)$ \\
\hline Fatigue & $7(11.3)$ \\
\hline Abdominal pain & $6(9.7)$ \\
\hline Weight loss & $6(9.7)$ \\
\hline Fever & $2(3.2)$ \\
\hline Jaundice & $1(1.6)$ \\
\hline Pruritus & $1(1.6)$ \\
\hline
\end{tabular}

involvement. Only 6 patients (9.7\%) were diagnosed with isolated hepatic sarcoidosis (Table 1).

Most patients were asymptomatic from liver disease ( $\mathrm{n}=35$; $56.5 \%$ ). Besides that, the most frequently reported symptom was fatigue, followed by abdominal pain, weight loss, fever, pruritus, and jaundice (Table 1 ).

A total of 9 patients in this cohort presented with clinically advanced hepatic involvement: cirrhosis was detected in 8 patients $(12.9 \%)$ at diagnosis of hepatic sarcoidosis, and 1 patient developed cirrhosis during follow-up of the study (1 year after diagnosis of hepatic sarcoidosis).

Moreover, $32.3 \%$ of patients $(n=20)$ with hepatic sarcoidosis had clinically significant portal hypertension. Clinical and radiological criteria used to diagnose portal hypertension were cross-sectional imaging (computed tomographic and/or magnetic resonance imaging) assessed by a hepatobiliary radiologist and endoscopic evaluation by a gastroenterologist. All 20 patients had radiological signs of portal hypertension (such as dilated portal vein, splenomegaly, and slow or reversed flow in portal veins), and 4 patients were found to have oesophageal varices on endoscopy. Interestingly, in $17.7 \%$ of patients $(n=11)$, portal hypertension was even seen in the absence of cirrhosis. Of those, 5 patients had severe portal fibrosis, and 6 patients $(9.7 \%)$ presented with portal hypertension without evidence of cirrhosis or fibrosis. Of our patients with hepatic sarcoidosis, $24.2 \%$ ( $\mathrm{n}=$ 15) already had portal hypertension at the time of diagnosis. In the remaining cases ( $8.1 \% ; n=5$ patients), it was diagnosed during follow-up of the study (after a median follow-up time of 7.4 years from initial diagnosis of hepatic sarcoidosis).

Hepatic events and complications of portal hypertension were exclusively observed in patients with cirrhosis. A single episode of hepatic encephalopathy (HE) was observed in 2 patients during follow-up of the study, which was reversible after therapy initiation. Moreover, 1 patient was diagnosed with hepatorenal syndrome (HRS) 7 years after the initial diagnosis of hepatic sarcoidosis, and 1 patient developed portal vein thrombosis during follow-up. Hydropic decompensation and oesophageal varices were detected in $6(9.7 \%)$ and $4(6.5 \%)$ patients, respectively. None of the patients underwent liver transplantation or developed hepatocellular carcinoma (HCC) during follow-up. Three of 9 patients diagnosed with cirrhosis died after a median time of 7.2 years from initial diagnosis of hepatic sarcoidosis: 1 patient suffered from variceal bleeding and died because of aspiration pneumonia during follow-up of the study, and 2 further patients with cirrhosis died during follow-up of acuteon-chronic liver failure (ACLF) owing to bacterial infections (spontaneous bacterial peritonitis [SBP] and urinary tract infection) as precipitating events.

\section{Clinical diagnostics}

At baseline, most patients presented with a cholestatic pattern of liver abnormalities: elevated alkaline phosphatase (ALP) and gamma glutamyl transferase (GGT) levels were the most prominent laboratory abnormalities observed in 41 (66.1\%) and 51 (82.3\%) patients, respectively. Mean serum ALP and GGT levels at baseline were 2.6 and 9.5 times above the upper limits of normal (ULNs) and were associated with mild elevations in transaminase levels (mean ALP: 272.1 IU/L; mean GGT: 381.4 IU/L; mean aspartate transaminase [AST]: $57.4 \mathrm{IU} / \mathrm{L}$; mean alanine transaminase [ALT]: $64.3 \mathrm{IU} / \mathrm{L}$; Table 2). Isolated or prominent elevation of transaminases was observed in none of the patients. Angiotensin-converting enzyme (ACE) and IL-2 receptor (IL-2R) levels were elevated in $19(57.6 \%)$ and 22 (66.7\%) of 33 patients,

\section{Table 2. Clinical diagnostics.}

\begin{tabular}{|c|c|c|}
\hline \multicolumn{3}{|l|}{ Clinical diagnostics } \\
\hline \multicolumn{3}{|l|}{ Liver biopsy, $\mathrm{n}(\%)$} \\
\hline \multicolumn{2}{|l|}{ Number of tested patients } & $55(88.7)$ \\
\hline \multicolumn{2}{|c|}{ Presence of noncaseating granuloma* } & $47(85.5)$ \\
\hline \multicolumn{3}{|c|}{ Imaging study at diagnosis of liver involvement, $\mathrm{n}(\%)^{\dagger}$} \\
\hline \multicolumn{2}{|c|}{ Number of tested patients } & $62(100)$ \\
\hline \multicolumn{2}{|l|}{ No abnormality } & $9(14.5)$ \\
\hline \multicolumn{2}{|l|}{ Hypodense nodular lesions } & $17(27.4)$ \\
\hline \multicolumn{2}{|l|}{ Hepatomegaly } & $16(25.8)$ \\
\hline \multicolumn{2}{|l|}{ Splenomegaly } & $16(25.8)$ \\
\hline \multicolumn{2}{|l|}{ Cirrhosis } & $9(14.5)$ \\
\hline \multicolumn{2}{|c|}{ Ascites } & $6(9.7)$ \\
\hline \multicolumn{3}{|c|}{ ACE at diagnosis of liver involvement, $n(\%)$} \\
\hline \multicolumn{2}{|c|}{ Number of tested patients } & $33(53.2)$ \\
\hline \multicolumn{2}{|c|}{ Number of patients with elevated ACE levels } & $19(57.6)$ \\
\hline $\begin{array}{l}\text { Liver biochemical tests } \\
\text { at diagnosis of liver involvement }\end{array}$ & Baseline & $\begin{array}{r}\text { Last } \\
\text { follow-up }\end{array}$ \\
\hline ALP (IU/L), mean \pm SD & $272.1 \pm 305.9$ & $149.2 \pm 147.3$ \\
\hline GGT $(\mathrm{IU} / \mathrm{L})$, mean $\pm \mathrm{SD}$ & $381.4 \pm 552.4$ & $229.4 \pm 448.3$ \\
\hline ALT (IU/L), mean \pm SD & $64.3 \pm 48.7$ & $43.8 \pm 31.7$ \\
\hline AST (IU/L), mean \pm SD & $57.4 \pm 49.2$ & $42.0 \pm 29.9$ \\
\hline Albumin (g/dl), mean \pm SD & $5.0 \pm 5.5$ & $4.1 \pm 0.9$ \\
\hline Total bilirubin $(\mathrm{mg} / \mathrm{dl})$, mean $\pm \mathrm{SD}$ & $1.02 \pm 0.90$ & $1.19 \pm 2.64$ \\
\hline Creatinine $(\mathrm{mg} / \mathrm{dl})$, mean $\pm \mathrm{SD}$ & $0.96 \pm 0.45$ & $0.95 \pm 0.43$ \\
\hline INR, mean \pm SD & $1.08 \pm 0.26$ & $1.1 \pm 0.2$ \\
\hline Platelets $(\mathrm{G} / \mathrm{L})$, mean $\pm \mathrm{SD}$ & $247.9 \pm 103.07$ & $220.8 \pm 81.1$ \\
\hline Cirrhosis, n (\%) & & $9(14.5)$ \\
\hline MELD score, mean \pm SD & & $9 \pm 3$ \\
\hline Child class A/B/C, n (\%) & & $7(11.3) / 2(3.2) / 0(0)$ \\
\hline
\end{tabular}

ACE, angiotensin-converting enzyme; ALP, alkaline phosphatase; ALT, alanine aminotransferase; AST, aspartate aminotransferase; GGT, gamma glutamyl transpeptidase; INR, international normalised ratio; MELD, model for end-stage liver disease.

* Presence of noncaseating granuloma among all tested patients.

More than 1 radiologic finding per patient was possible.

$\doteqdot$ Number of elevated ACE levels among all tested patients. 
whose tests were available at baseline. ALP levels were significantly correlated with IL-2R levels, but not with ACE levels at baseline and at the end of follow-up $(r=0.56, p=0.001 ; r=0.29$, $p=0.10)$.

Cholestatic liver parameters as well as transaminase levels significantly decreased at the end of follow-up compared with baseline (ALP: $p=0.002$; GGT: $p=0.001$; AST: $p=0.017$; ALT: $p=$ 0.009). Normalisation of ALP occurred in 16 of 41 patients with elevated levels at diagnosis, whereas 15 had lower levels but not within the normal range. Of the 16 patients with normalised ALP levels, a corresponding reduction in serum AST and ALT levels was observed in 14 and 15 patients, respectively. Of the 51 patients who had elevated GGT at baseline, 12 normalised by the end of follow-up, and 25 had lower values but not within the normal range. Moreover, $24.2 \%$ and $50.0 \%$ of patients had normal AST and ALT levels at the end of follow-up compared with 33.9\% and $27 \%$ at baseline, respectively. Hyperbilirubinemia was detected in 10 patients at baseline and in 5 patients at the end of follow-up. Moreover, 10 patients presented with thrombocytopaenia at baseline, of whom 4 were found to have splenomegaly. At the end of follow-up, thrombocytopaenia was found in 12 patients, of whom 8 had splenomegaly.

Imaging studies (abdominal ultrasound, computerised tomography, and magnetic resonance tomography) were performed for diagnostic evaluation in all patients. The most common radiographic finding were hypodense nodular lesions (17 cases), followed by hepatomegaly (16 cases) and splenomegaly (16 cases). Liver biopsy was performed in 55 patients. Noncaseating granulomas were found in 47 cases.

\section{Treatment}

An overview of all medical agents used in the study cohort including biochemical responses and discontinuations as a result of adverse effects or drug-induced hepatotoxicity is shown in Table 3.

A total of 27 patients were medically treated with a monotherapy, 27 received a combination of 2 or more agents during the follow-up period, whereas 8 patients were never treated medically. Oral glucocorticoids were the most frequently used in 19 patients (30.6\%) as monotherapy and in 24 patients (38.7\%) in combination with other medical agents. Follow-up was available in 35 patients. Mean change in ALP and ALT from baseline to 12-24 months after start of therapy was -168.0 and -37.0 (ALP: $p$ $<0.001$; ALT: $p=0.001$; Table 3), and normalisation was observed in 16 and 12 patients, respectively. A total of 25 patients with hepatic sarcoidosis were exposed to ursodeoxycholic acid (UDCA) at some point during follow-up, of whom 22 had available follow-up. A biochemical response was observed 12-24 months after start of therapy: ALP and ALT levels significantly decreased (ALP, mean change: $-219.7 ; p=0.003$; ALT, mean change: $-37.6, p=0.002$ ), and normalisation was achieved in $54.5 \%(\mathrm{n}=12)$ and $59.1 \%(\mathrm{n}=13)$ of UDC-treated patients, respectively. Moreover, improvement of liver function tests during therapy with corticosteroids and UDCA was often associated with a clinical response: $83.3 \%$ (10 of 12) and 75.0\% (9 of 12) of patients with normalised liver biochemistries under therapy with corticosteroids and UDCA, respectively, were asymptomatic at the end of follow-up compared with $50 \%$ and $41.7 \%$ patients at baseline. Regarding the clinical course of hepatic sarcoidosis, $75.0 \%(\mathrm{n}=9$ of 12$)$ and $91.7 \%(\mathrm{n}=11$ of 12$)$ of patients with normalised liver parameters under treatment with glucocorticoids and UDCA presented with a stable course of disease, respectively. Regression of sarcoidosis was observed in 4 patients with normalised liver biochemistries, of whom 3 were treated with corticosteroids and 1 was treated with UDCA.

Concerning tolerance to treatment, half of the patients (51.2\%) did not experience side effects on corticosteroids. The remaining patients reported side effects of varying severity $(20.9 \%$ of patients with deterioration of diabetic metabolism, 9.3\% of patients with osteopenia or osteoporosis, $4.7 \%$ of patients with new onset of arterial hypertension, $4.7 \%$ of patients with sleeping disorders, $4.7 \%$ of patients with nausea, $4.7 \%$ of patients with fatigue, and $4.7 \%$ of patients with myalgia). Few side effects were reported under therapy with UDCA: 2 of 25 patients suffered from gastrointestinal symptoms such as diarrhoea or nausea. All other patients were symptom-free.

Antimetabolites such as azathioprine (AZA) and methotrexate (MTX) were used in 11 and 9 patients, respectively. Improvement in liver parameters was observed in both therapy regimens (AZA, ALP: -98.2, ALT: +11.5; MTX, ALP: -126.9, ALT: -58.9). However, in contrast to treatments with steroids and UDCA, decreases in ALP and ALT activity were not observed to be significant (Table 3). Moreover, therapy with AZA needed to be stopped in 5 cases: 2 patients developed drug-induced hepatotoxicity, and 3 patients discontinued therapy prematurely owing to adverse effects. After stopping therapy with AZA owing to side effects, mycophenolate mofetil (MMF) was initiated in 1 patient, who achieved biochemical response 12 months after starting therapy with a normalisation of all liver parameters. Treatment with MTX needed to be stopped in 2 patients owing to drug-induced hepatotoxicity.

Regarding biologic agents, 2 patients were treated with cyclophosphamide and 1 patient with infliximab. In all 3 cases, decisions regarding treatment were primarily guided by the severity and activity of non-hepatic sarcoidosis, and improvement of liver parameters could not be detected.

\section{Discussion}

To our knowledge, our study represents the largest evaluation of epidemiology, clinical characteristics, and outcome of hepatic sarcoidosis. Compared with that in the cohorts of previous studies, which reported a prevalence of hepatic involvement by sarcoidosis between $5 \%$ and $30 \%$, the prevalence in this cohort was lower. ${ }^{11-13}$ The lower frequency is probably caused by the ethnic background of our study cohort, which is predominantly Caucasian. In fact, the risk of developing clinical manifestation of sarcoidosis and hepatic involvement varies across ethnic groups and is found to be 3 times more common among African Americans. Moreover, in our cohort, diagnosis of hepatic sarcoidosis relies on clinical, histopathological findings. Previous post-mortem data based on autopsy reported a considerably higher prevalence of hepatic sarcoidosis, suggesting that antemortem studies such as ours probably underestimate hepatic involvement in clinical practice. ${ }^{6}$ One reason for the discrepancy in prevalence of hepatic involvement between antemortem and post-mortem studies may be the relatively asymptomatic course of the disease. In line with previous studies, the majority of patients in our cohort did not have any symptoms, and only 27 patients were symptomatic, with fatigue being the most reported symptom. ${ }^{14,15}$ Cholestatic symptoms such as pruritus and jaundice were observed in only 1 patient, although the majority of patients had elevated cholestatic liver enzymes. Thus, most cases of hepatic sarcoidosis were not diagnosed by further 
Table 3. Efficacy and safety of medical agents used in patients with hepatic sarcoidosis.

\begin{tabular}{|c|c|c|c|c|c|c|c|c|c|c|}
\hline Medical drug & $\begin{array}{l}\text { Patients treated, } \\
\text { n }\end{array}$ & $\begin{array}{r}\text { Patients } \\
\text { responding, } \\
\mathbf{n}(\%)\end{array}$ & $\begin{array}{l}\text { Combi/ } \\
\text { monotherapy }\end{array}$ & $\begin{array}{r}\text { ALP pre-treatment } \\
(\mathrm{IU} / \mathrm{L}), \text { mean } \pm \mathrm{SD}\end{array}$ & $\begin{array}{l}\text { ALP post-treatment } \\
(\mathrm{IU} / \mathrm{L}), \text { mean } \pm \text { SD }\end{array}$ & $p$ & $\begin{array}{r}\text { ALT pre- } \\
\text { treatment }(\mathrm{IU} / \mathrm{L}), \\
\text { mean } \pm \mathrm{SD}\end{array}$ & $\begin{array}{r}\text { ALT post- } \\
\text { treatment }(\mathrm{IU} / \mathrm{L}), \\
\text { mean } \pm \mathrm{SD}\end{array}$ & $p$ & $\begin{array}{l}\text { Discontinuation } \\
\text { of } \\
\text { treatment as a } \\
\text { result of adverse } \\
\text { effects }\end{array}$ \\
\hline Corticosteroids & $\begin{array}{l}43 \text { patients treated } \\
\text { ( } 35 \text { patients with } \\
\text { available } F U \text { ) }\end{array}$ & $16(45.7)$ & $\begin{array}{l}24 \text { cases with } \\
\text { combitherapy } \\
\text { (UDCA or biologic } \\
\text { agents), } 19 \text { with } \\
\text { monotherapy }\end{array}$ & $276.2 \pm 218.3$ & $108.2 \pm 54.5$ & $<0.001$ & $76.2 \pm 54.4$ & $38.3 \pm 26.2$ & 0.001 & 0 \\
\hline UDCA & $\begin{array}{l}25 \text { patients treated } \\
\text { ( } 22 \text { patients with } \\
\text { available FU) }\end{array}$ & $12(54.5)$ & $\begin{array}{l}19 \text { cases with } \\
\text { combitherapy, } \\
6 \text { with } \\
\text { monotherapy }\end{array}$ & $366.4 \pm 440.9$ & $146.7 \pm 178.4$ & 0.003 & $75.6 \pm 56.7$ & $38.0 \pm 28.3$ & 0.002 & 0 \\
\hline Azathioprin & $\begin{array}{l}11 \text { patients treated } \\
\text { (11 with available } \\
\text { FU) }\end{array}$ & $5(45.5)$ & $\begin{array}{l}10 \text { cases with } \\
\text { combitherapy, } \\
1 \text { case with } \\
\text { monotherapy }\end{array}$ & $252.7 \pm 226.5$ & $154.5 \pm 97.2$ & 0.45 & $59.8 \pm 41.1$ & $71.3 \pm 93.8$ & 0.62 & $\begin{array}{l}2 \text { cases as a result } \\
\text { of drug-induced } \\
\text { hepatotoxicity and } \\
3 \text { cases as a result } \\
\text { of adverse effects }\end{array}$ \\
\hline Methotrexate & $\begin{array}{l}9 \text { patients treated } \\
\text { ( } 9 \text { with available } \\
\text { FU) }\end{array}$ & $3(33.3)$ & $\begin{array}{l}8 \text { cases with } \\
\text { combitherapy, } \\
1 \text { case with } \\
\text { monotherapy }\end{array}$ & $275.4 \pm 522.0$ & $148.5 \pm 119.4$ & 0.53 & $135.5 \pm 176.4$ & $76.6 \pm 79.9$ & 0.80 & $\begin{array}{r}2 \text { cases as a result } \\
\text { of drug-induced } \\
\text { hepatotoxicity }\end{array}$ \\
\hline MMF & $\begin{array}{l}1 \text { patient treated } \\
(1 \text { with available } \\
\text { FU) }\end{array}$ & $1(100)$ & $\begin{array}{l}1 \text { case with } \\
\text { combitherapy }\end{array}$ & 285.0 & 82.0 & 0.31 & 39.0 & 13.0 & 0.31 & 0 \\
\hline Cyclophosphamide & $\begin{array}{l}2 \text { patients treated } \\
(2 \text { with available } \\
\text { FU })\end{array}$ & $0(0)$ & $\begin{array}{l}1 \text { case with } \\
\text { combitherapy }\end{array}$ & $80.5 \pm 23.3$ & $93.0 \pm 2.8$ & 1.0 & $38.0 \pm 31.9$ & $30.0 \pm 26.8$ & 0.43 & n. a. \\
\hline Infliximab & $\begin{array}{l}1 \text { patient treated } \\
(1 \text { with available } \\
\mathrm{FU})\end{array}$ & $0(0)$ & $\begin{array}{l}1 \text { case with } \\
\text { combitherapy }\end{array}$ & 427.0 & 770.0 & 0.31 & 80.0 & 91.0 & 0.31 & n. a. \\
\hline
\end{tabular}


evaluation of symptoms but rather by patients presenting with elevated liver tests or radiologic imaging findings. Therefore, recommendation for routine screening of hepatic involvement in patients with sarcoidosis should be further evaluated.

In line with previous data, in our study, it was demonstrated that hepatic sarcoidosis is characterised by a cholestatic pattern of abnormalities, likely reflecting the infiltrative nature of the disease. Up to 55 and $73.3 \%$ of patients presented with ALP and GGT levels elevated more than 1.5 times over the ULN at baseline, whereas transaminase elevations were found to be less frequent and less severe. Moreover, none of the patients had isolated or prominent elevation of transaminases.

ACE levels were only elevated in $57.6 \%$ of tested patients, reflecting its poor sensitivity for diagnosing hepatic sarcoidosis, as it has already been reported for systemic sarcoidosis.

IL-2R, a parameter that has been shown to correlate with disease activity of sarcoidosis, was also elevated in only $66.7 \%$ of tested patients. However, in contrast to ACE, significant associations between IL-2R levels and cholestatic liver parameters could be detected, suggesting that these associations could reflect the degree of inflammation and therefore monitoring the clinical course of disease could be useful. ${ }^{16}$

Large randomised controlled studies on hepatic sarcoidosis are still missing. Thus, clear recommendations for the beginning and monitoring of therapy as well as data concerning the efficacy and long-term benefits of medical agents used are not available. Moreover, it is still unclear whether biochemical improvements alter sufficiently the long-term probability of liverrelated death or the need for transplantation. Glucocorticoids are often the first therapeutic approach in most forms of extrathoracic sarcoidosis. However, their use in hepatic sarcoidosis has only been evaluated in a few case studies till now. In our cohort, biochemical response to corticosteroids could be detected: 43 patients were treated with glucocorticoids, of whom 35 had available follow-up during therapy. Of those patients, $16(45.7 \%)$ responded well to treatment with normalisation of ALP and significant reduction of transaminases. Comparable results could be observed for treatment with UDCA. Of 25 patients receiving therapy, 22 had available follow-up presenting with significant reductions in all liver tests and normalised ALP in 12 cases (54.5\%). However, the clear endpoint of treatment is not the biochemical response but rather to control symptoms and to prevent disease progression to cirrhosis and portal hypertension. In that regard, improvement of clinical presentation was observed in most of the patients treated with UDCA or corticosteroids (63.6 and $77.8 \%$ of patients were asymptomatic at the end of follow-up compared with 40.9 and $47.2 \%$ of patients before therapy initiation, respectively). Regarding the clinical course of hepatic sarcoidosis, no significant benefits but a stable course of disease could be detected in the majority of patients, which probably shows that UDCA and corticosteroids may delay complications and progression of hepatic sarcoidosis.

Concerning antimetabolites, AZA and MTX were the medical agents used most frequently in this cohort. Experience with both drugs as treatment option in hepatic sarcoidosis is still limited. Kennedy et al. reported 2 and 3 cases of successful treatment with AZA and MTX. ${ }^{17}$ In our study, decisions regarding treatment with AZA and MTX were primarily guided by the severity and activity of non-hepatic sarcoidosis (AZA: $n=$ 5 patients with progredient pulmonary involvement of sarcoidosis; $\mathrm{n}=4$ patients with steroid refractory hepatic sarcoidosis; $\mathrm{n}=1$ patient with cardial manifestation; $\mathrm{n}=1$ patient with disseminated sarcoidal organ involvement; MTX: $\mathrm{n}=4$ patients with progressive pulmonal sarcoidosis, $\mathrm{n}=3$ patients with steroid refractory hepatic sarcoidosis, and $n=2$ patients with cutaneous and multiorgan involvement). Normalisation of ALP levels was detected in 5 of 11 patients treated with AZA and in 3 of 9 patients under treatment with MTX. However, limitations caused by side effects and hepatotoxicity were observed for both agents (AZA: 5 cases; MTX: 2 cases). Therefore, the use of antimetabolites should be evaluated critically in asymptomatic patients or patients with mild disease that may spontaneously remit.

Previous evidence suggested that liver involvement in sarcoidosis was common but the course of the disease tended to run mild with pulmonary involvement dominating the clinical picture. ${ }^{9}$ However, $12.9 \%$ (8 patients) of the entire cohort were found to have cirrhosis at the time of diagnosis with 1 more being diagnosed during follow-up. This frequency is similar to that of previous studies, which reported prevalence of cirrhosis caused by hepatic sarcoidosis ranging between 6 and 24\%. All of these patients had signs of portal hypertension, and 3 of them died because of liver-related complications during followup. Portal hypertension has rarely been reported complicating hepatic sarcoidosis. In our study, it was comparatively frequently found: $33.8 \%$ of patients with hepatic sarcoidosis had clinically significant portal hypertension. Interestingly, in $27.4 \%$ of patients, it was even seen without pathological evidence of cirrhosis. Portal hypertension in the absence of cirrhosis is not completely understood and may underlie different pathophysiological mechanisms. One hypothesis is that portal hypertension develops because of a presinusoidal block that is caused by portal granulomas. ${ }^{19,20}$ Further theories suggest that arterio-venous shunts, which form next to sarcoid granulomas, may lead to elevated portal flow resulting in an compensatory increase of intrahepatic resistance. ${ }^{21}$ It is very important to be aware that a considerable proportion of the patients with sarcoidosis has portal hypertension without evidence of cirrhosis, so histopathological exclusion of cirrhosis may not be able to rule out portal hypertension. Moreover, splenomegaly or ascites are not reliable indicators for portal hypertension and may also be caused by other factors in patients with hepatic sarcoidosis. We recommend cross-sectional imaging and endoscopic evaluation for every patient with hepatic sarcoidosis to rule out portal hypertension.

The frequent finding of advanced stages of liver disease in our study emphasises that liver involvement in sarcoidosis can be serious and life-threatening, independent of other organ involvement. Thus, routine laboratory evaluation for hepatic involvement should be performed in patients with sarcoidosis to prevent a delay in diagnosis. Moreover, patients diagnosed with hepatic sarcoidosis should be carefully monitored for progression and complications of the disease.

A major limitation of our study is its retrospective design. Diagnostic tests such as biochemical values or liver imaging studies as well as initiation or change of therapeutic regimens were at the physician's discretion and not inspired by a predefined study protocol. Thus, results of the study might be influenced by selection and surveillance bias. Moreover, our cohort is predominantly characterised by Caucasian individuals. Therefore, generalisability to other populations may be limited, given that the risk of developing clinical manifestation and complications of organ involvement by sarcoidosis varies across 
ethnic groups and is found to be 3 times more common among African Americans.

In summary, our multicentre, retrospective cohort emphasises the importance of correctly and early identifying hepatic involvement in sarcoidosis, because of the potentially progressive and complicated course of disease.

The relatively asymptomatic nature of hepatic sarcoidosis, which was observed in the majority of the cohort, could be responsible for the discrepancy in prevalence estimates as well as for the delay in diagnosis and treatment of many patients. Thus, routine screening of hepatic involvement in patients with sarcoidosis should be further discussed. Biochemically, a cholestatic pattern of liver enzyme elevations was the most common abnormality. Regarding pharmacological treatment options, corticosteroids and UDCA were the medical agents most frequently used, and both of them have been shown to induce biochemical response in the majority of patients. Therefore, corticosteroids and UDCA should be used as primary treatment modality for symptomatic patients with abnormal liver function tests. Secondand third-line treatment options should only be considered in cases of disease worsening as a result of their potential hepatotoxicity and side effects. Future prospective studies are warranted to further evaluate the effect of therapy especially on clinically relevant endpoints such as progression to cirrhosis or liver-related death.

\begin{abstract}
Abbreviations
ACE, angiotensin-converting enzyme; ACLF, acute-on-chronic liver failure; ALP, alkaline phosphatase; ALT, alanine transaminase; AST, aspartate transaminase; ATS, American Thoracic Society; AZA, azathioprine; GGT, gamma glutamyl transferase; HCC, hepatocellular carcinoma; HE, hepatic encephalopathy; HRS, hepatorenal syndrome; ICD-10, International Classification of Diseases, Tenth Revision; IL-2R, IL-2 receptor; MMF, mycophenolatmofetil; MTX, methotrexate; SBP, spontaneous bacterial peritonitis; ULN, upper limit of normal; UDCA, ursodeoxycholic acid.
\end{abstract}

\section{Financial support}

The authors received no financial support to produce this manuscript.

\section{Conflicts of interest}

CG has received travel support from Gilead and speaking fees from AbbVie outside the submitted work. JMS is a consultant at BMS, Boehringer Ingelheim, Echosens, Genfit, Gilead Sciences, Intercept Pharmaceuticals, Madrigal, Nordic Bioscience, Novartis, Pfizer, Roche, Sanofi, and Siemens Healthcare GmbH. JMS also has received research funding from Gilead Sciences, Boehringer Ingelheim, and Siemens Healthcare GmbH. JMS has received speakers' honoraria from Falk Foundation and MSD Sharp \& Dohme GmbH all outside of the submitted work. CML has received speaker and consultancy fees outside the present work from AbbVie, Gilead, Falk, MSD, Novartis, Roche, and Eisai. JD has received research support from Gilead outside the submitted work. JV has received speaking and/or consulting fees from Abbott, AbbVie, Bristol-Myers Squibb, Gilead, Medtronic, Merck/MSD, and Roche outside the submitted work. SZ has received speaking and/or consulting fees from AbbVie, Bristol-Myers Squibb, Falk, Gilead, Janssen, and Merck/MSD outside the submitted work. AP has received travel support from AbbVie and BMS and lecturer fees from AbbVie, BMS, and Gilead. All other authors declare no competing interests.

Please refer to the accompanying ICMJE disclosure forms for further details.

\section{Authors' contributions}

Design of the study concept: CG, AP. Data collection: JA, CML, KW, JMS, JS, JLM, TB, JD, NW, AM. Analysis and interpretation of data: CG, AP. Draft of the manuscript: CG. Critical revision of the manuscript: JV, CML, JMS, JS, $\mathrm{GR}, \mathrm{SZ}, \mathrm{AP}$.

\section{Data availability statement}

The raw data supporting the conclusions of this article will be made available by the authors, without undue reservation, to any qualified researcher.

\section{Supplementary data}

Supplementary data to this article can be found online at https://doi.org/1 0.1016/j.jhepr.2021.100360.

\section{References}

[1] Tadros M, Forouhar F, Wu GY. Hepatic sarcoidosis. J Clin Transl Hepatol 2013:1:87-93.
[2] Baughman RP, Lower EE, du Bois RM. Sarcoidosis. Lancet 2003;361:11111118.

[3] Iannuzzi MC, Rybicki BA, Teirstein AS. Sarcoidosis. N Engl J Med 2007;357:2153-2165.

[4] Grunewald J, Grutters JC, Arkema EV, Saketkoo LA, Moller DR, MüllerQuernheim J. Sarcoidosis. Nat Rev Dis Prim 2019;5:1-22.

[5] Ghanim MT, Tiwari A, Nawras A. Hepatic sarcoidosis suspected for liver cirrhosis-a case report of diagnosing subclinical disease: 2210. Am J Gastroenterol 2018;113:S1256.

[6] Iwai K, Oka H. Sarcoidosis. Report of ten autopsy cases in Japan. Am Rev Respir Dis 1964;90:612-622.

[7] Hamesch K, Mandorfer M, Pereira VM, Moeller LS, Pons M, Dolman GE et al. Liver fibrosis and metabolic alterations in adults with alpha-1antitrypsin deficiency caused by the Pi*ZZ mutation. Gastroenterology 2019;157:705-719.e18.

[8] Ala A, Walker AP, Ashkan K, Dooley JS, Schilsky ML. Wilson's disease. Lancet 2007;369:397-408.

[9] Kumar M, Herrera JL. Sarcoidosis and the liver. Clin Liver Dis $2019 ; 23: 331-343$.

[10] Crouser ED, Maier LA, Wilson KC, Bonham CA, Morgenthau AS, Patterson KC, et al. Diagnosis and detection of sarcoidosis. An official American Thoracic Society clinical practice guideline. Am J Respir Crit Care Med 2020;201:e26-e51.

[11] Judson MA, Boan AD, Lackland DT. The clinical course of sarcoidosis: presentation, diagnosis, and treatment in a large white and black cohort in the United States. Sarcoidosis Vasc Diffuse Lung Dis 2012;29:119-127.

[12] Cozier YC, Berman JS, Palmer JR, Boggs DA, Serlin DM, Rosenberg L. Sarcoidosis in black women in the United States: data from the black women's health study. Chest 2011;139:144-150.

[13] Bihari C, Rastogi A, Kumar N, Rajesh S, Sarin SK. Hepatic sarcoidosis: clinico-pathological characterization of symptomatic cases. Acta Gastroenterol Belg 2015;78:306-313.

[14] Devaney K, Goodman ZD, Epstein MS, Zimmerman HJ, Ishak KG. Hepatic sarcoidosis. Clinicopathologic features in 100 patients. Am J Surg Pathol 1993; 17:1272-1280.

[15] Ungprasert P, Carmona EM, Crowson CS, Matteson EL. Diagnostic utility of angiotensin converting enzyme in sarcoidosis: a population-based study. Lung 2016;194:91-95.

[16] Kennedy PTF, Zakaria N, Modawi SB, Papadopoulou AM, Murray-Lyon I, du Bois RM, et al. Natural history of hepatic sarcoidosis and its response to treatment. Eur J Gastroenterol Hepatol 2006;18:721-726.

[17] Judson MA. Extrapulmonary sarcoidosis. Semin Respir Crit Care Med 2007;28:83-101.

[18] Blich M, Edoute Y. Clinical manifestations of sarcoid liver disease. J Gastroenterol Hepatol 2004;19:732-737.

[19] Judson MA. Hepatic, splenic, and gastrointestinal involvement with sarcoidosis. Semin Respir Crit Care Med 2002;23:529-541.

[20] Sedki M, Fonseca N, Santiago P, Diaz L, Garcia-Buitrago M, Mirsaeidi M, et al. Hepatic sarcoidosis: natural history and management implications. Front Med (Lausanne) 2019;6:232.

[21] Maddrey WC, Johns CJ, Boitnott JK, Iber FL. Sarcoidosis and chronic hepatic disease: a clinical and pathologic study of 20 patients. Medicine (Baltimore) 1970;49:375-395. 